\title{
Veterans Are Agreeable to Discussions About Firearms Safety in Primary Care
}

\author{
Summer Newell, PhD, MPH, Emily Kenyon, BA, Khaya D. Clark, PhD, \\ Victoria Elliott, MscPH, Annabelle Rynerson, BS, Martha S. Gerrity, MD, \\ Elizabeth Karras, PhD, Joseph A. Simonetti, MD, MPH, and Steven K. Dobscha, MD
}

Background: Discussing safe storage of firearms, including access, during times of crisis with veterans in primary care settings may enhance suicide prevention efforts. However, veteran attitudes toward such discussions are not well understood. The goal of this study is to understand the perspectives of veterans on discussing firearms storage safety with staff during primary care visits.

Methods: Individual semistructured interviews with veterans were conducted by telephone, qualitatively coded, and analyzed for themes. The sample was composed of veterans $(n=27)$ who had positive depression or post-traumatic stress disorder screens and who received care from Veterans Health Administration primary care team members trained to discuss firearms storage safety with patients.

Results: Citing the urgent need to prevent veteran suicide, most veterans felt discussing firearms safety was acceptable and needed, even if discussions felt uncomfortable or they had concerns.

Veterans identified the need for providers to be transparent in their purposes for asking about firearms and to respect veterans' unique relationships with firearms.

Discussion: Conducting firearms safety discussions in a primary care setting with veterans who are at elevated risk for suicide is acceptable to veterans when a respectful, veteran-centered, and transparent approach is used. ( $\mathrm{J}$ Am Board Fam Med 2021;34:338-345.)

Keywords: Depression, Firearms, Interview, Mental Health, Military Medicine, Post-Traumatic Stress Disorders, Primary Health Care, Qualitative Research, Suicide, Veterans Health

\section{Introduction}

Suicide risk is higher among veterans than age and sex-matched nonveteran adults, ${ }^{1}$ and veteran suicide prevention is a leading national priority. Firearms are used in half of suicides in the United

This article was externally peer reviewed.

Submitted 27 May 2020; revised 30 September 2020; accepted 1 October 2020.

From the Center to Improve Veteran Involvement in Care (CIVIC), VA Portland Health Care System, Portland, OR (SN, EKenyon, VE, AR, SKD); Division of Psychology, Oregon Health \& Science University, Portland (EKenyon); Department of Medical Informatics and Clinical Epidemiology, Oregon Health \& Science University, Portland, OR (KDC); National Center for Rehabilitative Auditory Research, VA Portland Health Care System, Portland, OR (KDC); Hearing Center of Excellence, Department of Defense, San Antonio, TX (KDC); Division of Hospital and Specialty Medicine, VA Portland Health Care System, Portland, OR (MSG); Division of General Internal Medicine and Geriatrics, Department of Medicine, Oregon Health \& Science University, Portland (MSG); Center of Excellence for Suicide Prevention, Department of Veterans Affairs, Canandaigua, NY (EKarras); Department of Psychiatry, University of Rochester Medical Center, Rochester, NY (EKarras); Rocky Mountain Mental Illness, Research, Education
States ${ }^{2}$; however, in 2017, 69\% of suicides among veterans were firearm related. ${ }^{1}$ Reducing access to lethal means is one of a few empirically supported approaches for decreasing suicide rates, ${ }^{3,4}$ and safe firearm storage practices have been associated with reduced risk of death by suicide. ${ }^{5,6}$ Counseling

and Clinical Center (MIRECC) for Suicide Prevention, VHA, Aurora, CO. (JAS); Denver-Seattle Center of Innovation for Veteran-Centered and Value-Driven Care, VHA, Aurora, CO (JAS); Department of Psychiatry, Oregon Health \& Science University, Portland (SKD).

Disclaimer: The views expressed in this article are those of the authors and do not necessarily reflect the position or policy of the Department of Veterans Affairs or United States government.

Conflict of interest: None declared.

Funding: This work was supported by the United States Department of Veterans Affairs (VA), Veterans Health Administration, VA Office of Mental Health and Suicide Prevention, and VA Health Services Research and Development Center to Improve Veteran Involvement in Care (CIVIC) (CIN 13-404).

Corresponding author: Summer Newell, PhD, MPH, VA Portland Health Care System, Portland, OR 97239 (E-mail: summer.newell@va.gov). 
approaches focused on reducing access to lethal means (ie, primarily firearms) have been developed in an effort to reduce deaths by suicide, ${ }^{7,8}$ and promising models and guidelines exist for delivering means safety counseling. ${ }^{7,8}$ Although means safety counseling can be delivered in any clinical setting, the knowledge and skills required to deliver that counseling have typically only been included in training for mental health clinicians. Yet, half of patients, including veterans, who die by suicide are seen in primary care settings in the month before death. ${ }^{9,10}$ Furthermore, most veterans, including those who die by suicide, access care outside of the Veterans Health Administration (VHA). ${ }^{11}$ These findings suggest that primary care clinics in public and private settings may be critical locations to engage veterans in discussions about firearm safety.

Many health systems have recognized the significant role of primary care in suicide prevention and have implemented screening protocols to identify patients with undiagnosed mental health conditions and/or elevated suicide risk, ultimately leading to mental health treatment and suicide risk mitigation strategies. Ideally, such strategies should include evidence-based interventions to reduce access to firearms among individuals with elevated suicide risk. In this article, we refer to discussions about safe firearms storage and decreasing access to firearms during times of elevated risk as firearm safety storage discussions. Safe firearms storage requires, at a minimum, keeping firearms locked and unloaded when not in use.

Studies have shown that veterans, ${ }^{12}$ other patient groups, ${ }^{13-15}$ and clinicians ${ }^{12,16}$ are frequently open to discussing firearms safety, especially when the information is delivered in a culturally sensitive, nonjudgmental manner ${ }^{17-19}$ and patients are made aware of the direct relevance of safe firearm storage to safety and well-being. ${ }^{20}$ Other studies suggest that the credibility of physicians as messengers regarding firearms safety may be limited. ${ }^{21-23}$ Recently published work $^{24,25}$ indicates veterans may have unique attitudes and experiences related to firearms that may impact firearms safety discussions with providers, and that trust and rapport between patients and providers is an important pretext for acceptable firearm discussions. However, the perspectives of patients, particularly veterans, on discussing firearms safety with staff during primary care visits are unknown. We therefore sought to identify the perspectives of veterans who screened positive for mental disorders associated with suicide risk (post-traumatic stress disorder [PTSD] and/or depression ${ }^{26}$ ) and had recently visited a clinician who had engaged in a training program on discussing firearms storage safety in primary care. A secondary objective was to identify, from the veteran perspective, best practices for talking about firearms storage safety during primary care visits.

\section{Methods}

Data used in the current analysis were derived from a larger project to evaluate the feasibility and acceptability of a novel program to train primary care staff to have firearms storage safety discussions in primary care settings. The training program focused primarily on equipping staff with background information and strategies for discussing safe storage of firearms with patients who likely have elevated risk for suicide, in this case, veterans who screen positive for depression or PTSD during routine primary care screenings. In the initial phase of the project, the research team used an iterative design process involving input from veterans and primary care staff to adapt and refine training content for use within VA primary care settings.

In the second phase of the project, we conducted a preliminary evaluation of the firearm storage safety training program. As one aspect of this effort, we gathered preliminary qualitative data (reported here) from patients to learn to what extent the education program may have impacted staff-patient interactions following depression and PTSD screening. Based on our and others' work showing low rates of documentation of firearms discussions in clinical records, even among patients with known elevated suicide risk, ${ }^{27-30}$ we expected that despite staff participation in the training program, many patients would not have had firearms safety discussions with their clinicians. We therefore also sought to gather patient perspectives on the idea of discussing firearms safety with primary care staff even when they had not had such discussions. The project was approved by the Institutional Review Board of VA Portland Health Care System.

\section{Setting and Participants}

This training and evaluation project took place within the VA Portland Health Care System, a 
large medical center in the Pacific Northwest that provides health care services to approximately 100,000 unique patients each year across 11 urban and rural clinics. The VHA national patient care database, a repository of clinical and administrative data for all VHA patients refreshed daily, was used to identify potentially eligible patients who (1) were seen by a primary care provider in the month following the staff member's participation in the firearms safety training program, and (2) had screened positive for depression (cutoff score of 3 on the Patient Health Questionnaire-2 [PHQ-2]) ${ }^{31}$ or PTSD (cutoff score of 4 on the primary care PTSD screen for the Diagnostic and Statistical Manual of Mental Disorders 5 [DSM-5] [PC-PTSD-5] $)^{32}$ during the primary care visit. It is standard practice for patients seen in VA primary care to also be administered a single item at the time of depression or PTSD screening that assesses for current suicidal thoughts (PHQ-9 item 9; thoughts of death or self-injury within the past 2 weeks). The suicidal thoughts screen result did not impact study eligibility. We excluded patients who had documented diagnoses in the electronic health record (EHR) of dementia, cognitive disturbance, or severe hearing impairment, which might impede participation in a telephone interview.

\section{Procedures}

Between April and August 2019, 1036 patients from the 2 VA Portland Health Care System clinics were assessed for potential eligibility using data from the EHR, and 382 letters were mailed introducing the study to those who met initial inclusion criteria and asking them to contact the research team if they would be willing to participate. The team followed up with a phone call 2 weeks later to veterans who did not respond to the letter. Twenty-seven patients were enrolled; 5 veterans contacted the team following receipt of the recruitment letter, and 22 were recruited via telephone follow-up. Participants completed an approximately 30-minute-long interview about their recent primary care visit. Verbal informed consent was obtained from all participants before interview participation. Each veteran received a $\$ 25$ gift card for completing the interview. Interviews were transcribed and coded by 3 members of the research team.
Interviews were conducted by telephone with experienced qualitative interviewers using an interview guide. During interviews, participants were asked if they recalled whether firearm safety was discussed at their recent primary care visit, their comfort with the conversation, and any thoughts regarding what their primary care team should know about when discussing firearm safety and mental health with veterans. For veterans who reported that they did not recollect discussing firearms safety at a primary care visit, we asked how they might feel if they were to be asked these questions by their provider.

We employed a hybrid conventional and directed content analysis to organize data into themes, ${ }^{33}$ first broadly seeking the pertinent perspectives of the interviewees. Next, to ensure that the projects' objectives were addressed, we followed with a directed analysis of themes such as "mental health in primary care" to complement the inductive approach. All 3 members of the coding team began by independently coding 3 (10\% of the sample) of the same interviews, followed by discussions to establish a shared understanding of meaning and to develop the codebook. Two members of the team independently coded each of the remaining 24 interviews, intermittently checking in with the coding team to discuss meanings and subsequent adaptations of the codebook. Feedback on themes was also sought from the broader author team. We used Atlas.ti software for all analyses. ${ }^{34}$ Demographic information was collected from the EHR or from self-report by the participants at the time of interviews.

\section{Findings}

Twenty-seven participants completed individual, semistructured interviews. Participants were mostly male (93\%), non-Hispanic white $(67 \%)$, and had an average age of 56 (range 27 to 81 ), which is similar to the veteran population overall. ${ }^{35}$ Veterans were diverse across armed service branches and service era: 10 served in the Army, 6 in the Navy, 7 in the Marine Corps, 5 in the Air Force, and 1 in the Air National Guard. Ten served post-9/11, 6 served during the Gulf War, and 12 served during the Vietnam era. Six participants (22\%) completed a 4year college degree or higher. Of those interviewed, $10(37 \%)$ recalled having had a firearms safety discussion. Personal firearm ownership was not 
explicitly assessed due to the sensitive nature of the topic. The literature suggests that approximately half of veterans own firearms. ${ }^{36}$ During our interviews, the majority of participants shared positive attitudes toward firearms ownership or self-disclosed ownership without prompting.

\section{Firearms Safety Discussions Are Necessary Given Suicide Risk among Veterans}

Nearly all veterans in the sample felt that firearms safe storage discussions were acceptable. The group consistently reported, without directly being asked, that they are aware of the current suicide crisis among fellow veterans. Despite any discomforts they might have, these veterans felt firearms discussions are appropriate and necessary given elevated rates of veteran suicides. When asked how they felt when asked about firearm storage by their primary care provider, a veteran explained that the questions may feel personal, but he understands why the VA is asking.

It doesn't bother me coming from them, because I trust them. It seems to me, it's nobody's business but mine. Then again, I also understand we're losing a bunch of guys every day to suicide...so I understand the question, beyond my personal feelings. $-506 \mathrm{a}$ (Vietnam era)

Given his concerns about the high suicide risk for post-9/11 veterans, another respondent described why asking about firearm storage safety in primary care is needed:

I feel like it's appropriate. I don't feel like it's inappropriate, considering what has happened in these past years. In fact, after 9/11, I had said to some of my family members, "there's going to be a lot of people that need a lot of health care. Not just physical, but there's going to be a lot of emotional issues coming out of this," and that was 18 years ago. -565 a (post-9/11 era)

Some veterans associated their own mental health experiences with their willingness to discuss firearm storage safety. One veteran who experiences suicidal ideation explained:

I've been asked that multiple times. Especially because I have severe suicidal ideation... it is a constant struggle, and firearms would make that very easy for me. I don't mind [being asked]. I'm fine with that. $-584 \mathrm{a}$ (post-9/11 era)

Despite general agreement with the rationale for firearms safety discussions, some veterans felt it was only appropriate to ask about firearm safety when risk indicators exist, rather than asking everyone.
One veteran who experienced some mental health concerns said:

My personal opinion on it would be, if a mental condition is established or there are strong depression indicators, then firearms questions should be asked at that point. Prior to that, I'm not sure it's relevant until there's an indicator that it's a concern. Which in my case, it was a concern. Asking me was a perfectly reasonable and logical thing to do. $-584 \mathrm{a}$ (post-9/11 era)

\section{Willingness to Discuss Firearms Safety Comes with Concerns}

Some veterans voiced concerns about the implications of disclosure in a clinical environment. Some reported they were worried the provider may want to remove their firearms or felt their provider might not understand their perspective on firearms.

It's a personal thing. I don't generally talk about it, because a lot of people don't understand it. 579a (Vietnam era)

Another veteran explained that being asked about firearms made him feel targeted as a gun owner, but that providing a rationale for the discussion may help ease those concerns.

I've been around firearms my whole life. I was in the military too. I don't see myself as a dangerous person, I don't consider myself that. But then you feel like you're being targeted because of it. Because you do everything you're supposed to, but then why are you asking me about somethingother people's issues become yours, you know?... Maybe more lead into the question, for some other people, might be less off-putting. [Not] Just, "you own firearms?" [laughs], maybe an explanation of the rationale. $-592 \mathrm{a}$ (post-9/11 era)

For a few veterans in our sample, talking about firearm safety in primary care did not "feel right." One veteran explained that firearm safety discussions should not be a priority for his primary care provider.

It would be like them portraying themselves as a cop [laughs]. I mean that's my outlook on it. It kind of pulls them away from being a doctor... like I say she's more focused on me as a person and not worrying about if I'm dealing with weapons. -783 b (Gulf War era)

\section{Veterans Have Unique Relationships to Firearms}

To understand some of the misgivings about providers discussing firearms in a health care setting, veterans reported that clinicians need to appreciate that military experiences may shape veterans' 
perspectives on firearm safety, and that these military experiences should be acknowledged within the context of discussing firearm safety.

I'm not sure that's an appropriate thing to ask [in primary care]—we're veterans. We worked around firearms our entire career. It's second nature to us. It's like asking an accountant "do you know how to work a calculator?" Which is a little insulting. It just is. -584 a (post-9/11 era)

One respondent believes veterans may take pause before disclosing information about their firearms to those who have not been in the military, including health care providers, because they may not share their perceptions of what it means to own and use firearms.

There are a lot of vets...it would seem like an invasion of privacy for people to be asking about them. It's like giving up your fighting position in a combat situation. It seems counterintuitive. I think [asking about firearm storage is] appropriate, although I would totally understand for any reason, no matter how nicely you say it, that there's no way that you can prevent some veterans from taking offense or being hesitant. $-568 \mathrm{a}$ (post-9/11 era)

To respect veterans' knowledge of firearms, one participant pointed to the importance of language. Specifically, clinicians should indicate they are concerned about firearm storage rather than using generic terms such as "firearm safety," because it should be obvious that the veteran understands firearm safety.

\section{Mixed Findings on How Veterans Want Firearm Safety Discussions Delivered}

Most veterans in our sample preferred personalized discussions of means safety with a primary care provider that they trust. However, for some veterans, a standardized and impersonal approach might feel more comfortable. One participant remembers being asked on a computer before his appointment, and explains why it was preferable:

I think when someone asks you that personally, and you're in that environment you immediately start thinking, "oh wait a minute, is there something wrong with me? Is there something that's causing them to ask that question?" That makes it feel a little awkward. -593 a (post-9/11 era)

\section{Discussion}

Nearly all participants in our study reported that they understood why a primary care provider might engage in firearms safety discussions, particularly given the elevation in suicide rates among veterans. Some misgivings were reported, with concerns that questions felt too personal or the provider's motives for asking about firearms were unclear (ie, whether firearms might be removed), which may reflect the documented division found between gun owners and nonowners where some gun owners are weary of gun control efforts. ${ }^{37}$ Yet, these misgivings did not supersede their agreeing to participating in firearms safety discussions, pointing to the strong potential for primary care staff to play a significant role in risk reduction.

Our interview findings also underscore the unique relationship and experiences veterans have with firearms, the impact of military service on their perspectives, and the need for this to be acknowledged and affirmed by clinicians during firearms discussions, which closely parallels recent findings by Simonetti et al. ${ }^{25}$ Our findings also align with other prior literature that suggests that, if done in a respectful and transparent manner, patients are comfortable discussing firearms safety in clinical settings, ${ }^{12,17,18}$ including those receiving mental health care. ${ }^{38}$ Contextualizing the question (ie, "firearm storage" is preferred over "firearm safety") as well as relating that questions are for the purpose of suicide prevention have also been recognized as helpful strategies. ${ }^{25}$ However, participants also described veteran-specific concerns of which primary care providers and teams should be aware, including recognition of veterans' history, relationship, and expertise with firearms. Notably, while the majority of participants preferred personalized discussions with providers, a few preferred that structured firearm safety questions be used, such that everyone be asked in the same, less personal way. The perspective of these few veterans aligns with prior research showing that structured, computerized methods for assessing and addressing sensitive behavior may be more appropriate for some individuals. ${ }^{39}$

Our findings have other implications for practice. Veterans are generally aware of elevated suicide risk among veterans; this information helps support the rationale for firearms storage safety discussions. In addition, acknowledging veterans' prior training and experience with firearms may be a way to convey respect. Taking advantage of these findings, a clinician might say, "I know you have been trained in the use of firearms, but because we know 
veterans have increased risk for suicide, I would like to have a conversation about your firearms. Is that OK?" Clearly, additional research needs to be conducted to identify and test potential scripts for clinicians to use to optimize acceptability and positive impact of firearms storage safety discussions. Our data also suggest that messaging about firearms safety may sometimes be more impactful if it comes from a staff member (eg, nurse or medical assistant) other than the primary care provider; to enhance credibility or help allay concerns about motives for the discussion, for example, by team members who are firearms owners or are veterans themselves. Finally, we note that many veterans do not receive VHA care, and some may not routinely receive any health care; thus, it is important to continue to develop multifaceted approaches to improving firearms safety that extend beyond the VHA and health care settings.

\section{Limitations}

Although our small, qualitative sample demographically resembles the larger veteran population, the study is subject to some selection and response biases in that our participants may have been more willing to talk about firearms. We note that our sample had a slightly higher average level of education compared with national norms for veterans. ${ }^{40}$ Veterans were also recruited from 2 suburban primary care clinics in the Portland. Oregon metropolitan area, which may not reflect perspectives of those in rural locations or in other parts of the country. As with the general population, veterans hold a broad range of sociopolitical views that may influence perceptions and communications about firearms ${ }^{41}$ that may not be fully represented here. Furthermore, while a number of suicide decedents have no known mental health diagnosis at time of death, ${ }^{42,43}$ our study garnered perspectives from veterans who screened positive for mental health conditions, pointing to the need for more extensive exploration with a larger, more diverse sample, including veterans not using VHA care. Finally, this study was exploratory; while only a few veterans in the sample recalled having spoken with a provider about firearms, attitudes around hypothetical future firearms safety discussions in primary care were explored. Future research is needed to tease out the nuances of the effects of staff training on patient outcomes.

\section{Conclusion}

More than half of veterans who die by suicide visit their primary care provider a month before their death, underscoring the critical role of primary care providers in suicide prevention. Our findings support that discussing firearm-related safety in such settings may be an acceptable practice among veterans. Specifically, the findings support discussions of firearms safety in VHA primary care settings as part of an effort to reach at-risk veteran populations who might not otherwise be identified for intervention and offer several lessons learned that may translate into practice. Participants were generally aware and concerned that veterans are at higher risk for suicide than other populations. This provides a veteran-specific rationale for firearm safety discussions. However, providers should be mindful of the need to conduct such discussions in a transparent and respectful manner that considers the unique relationship veterans have with firearms.

To see this article online, please go to: bttp://jabfm.org/content/ 34/2/338.full.

\section{References}

1. Office of Mental Health and Suicide Prevention. 2019 National Veteran Suicide Prevention Annual Report. 2019.

2. Centers for Disease Control and Prevention (CDC). Web-based Injury Statistics Query and Reporting System (WISQARS). Available from: https://wisqars-viz.cdc.gov/. Accessed April 3, 2018.

3. Mann JJ, Apter A, Bertolote J, et al. Suicide prevention strategies: a systematic review. JAMA 2005; 294:2064-74.

4. Bagley SC, Munjas B, Shekelle P. A systematic review of suicide prevention programs for military or veterans. Suicide Life Threat Behav 2010;40: 257-65.

5. Shenassa ED, Rogers ML, Spalding KL, Roberts MB. Safer storage of firearms at home and risk of suicide: a study of protective factors in a nationally representative sample. J Epidemiol Community Health 2004;58:841-8.

6. Dempsey CL, Benedek DM, Zuromski KL, et al. Association of firearm ownership, use, accessibility, and storage practices with suicide risk among US Army soldiers. JAMA Netw Open 2019;2:e195383.

7. Suicide Prevention Resource Center. CALM: Counseling on Access to Lethal Means online course. Available from: http://www.sprc.org/resourcesprograms/calm-counseling-access-lethal-means. Accessed March 8, 2018.

8. Means Matter, Harvard T.H. Chan School of Public Health. Lethal means counseling: recommendations 
for clinicians. Available from: https://www.hsph. harvard.edu/means-matter/recommendations/ clinicians/. Accessed March 8, 2018.

9. Denneson LM, Kovas AE, Britton PC, Kaplan MS, McFarland BH, Dobscha SK. Suicide risk documented during veterans' last Veterans Affairs health care contacts prior to suicide. Suicide Life Threat Behav 2016;46:363-74.

10. Luoma JB, Martin CE, Pearson JL. Contact with mental health and primary care providers before suicide: a review of the evidence. Am J Psychiatry 2002;159:909-16.

11. Hogan M. Veteran suicide: not just a VA issue; it's a U.S. issue. Ann Intern Med 2019;171:372-3.

12. Walters H, Kulkarni M, Forman J, Roeder K, Travis J, Valenstein M. Feasibility and acceptability of interventions to delay gun access in VA mental health settings. Gen Hosp Psychiatry 2012;34:692-8.

13. Betz ME, Miller M, Barber C, et al. Lethal means access and assessment among suicidal emergency department patients. Depress Anxiety 2016;33: $502-11$.

14. Betz ME, Azrael D, Johnson RL, et al. Views on firearm safety among caregivers of people with Alzheimer disease and related dementias. JAMA Netw Open 2020;3:e207756.

15. Boge LA, Dos Santos C, Burkholder JD, Koschel BR, Cubeddu LX, Farcy DA. Patients' perceptions of the role of physicians in questioning and education in firearms safety: post-FOPA repeal era. South Med J 2019;112:34-8.

16. Cassel CK, Nelson EA, Smith TW, Schwab CW, Barlow B, Gary NE. Internists' and surgeons' attitudes toward guns and firearm injury prevention. Ann Intern Med 1998;128:224-30.

17. Marino E, Wolsko C, Keys S, Wilcox H. Addressing the cultural challenges of firearm restriction in suicide prevention: a test of public health messaging to protect those at risk. Arch Suicide Res 2017;1-11.

18. Stanley IH, Hom MA, Rogers ML, Anestis MD, Joiner TE. Discussing firearm ownership and access as part of suicide risk assessment and prevention: "means safety" versus "means restriction." Arch Suicide Res 2017;21:237-53.

19. Betz ME, Wintemute GJ. Physician counseling on firearm safety: a new kind of cultural competence. JAMA 2015;314:449-50.

20. Sege RD, Hatmaker-Flanigan E, De Vos E, Levin-Goodman R, Spivak H. Anticipatory guidance and violence prevention: results from family and pediatrician focus groups. Pediatrics 2006; 117:455-63.

21. Crifasi CK, Doucette ML, McGinty EE, Webster DW, Barry CL. Storage practices of US gun owners in 2016. Am J Public Health 2018;108:532-7.
22. Knoepke CE, Allen A, Ranney ML, Wintemute GJ, Matlock DD, Betz ME. Loaded questions: internet commenters' opinions on physician-patient firearm safety conversations. West J Emerg Med 2017; 18:903-12.

23. Shaughnessy AF, Cincotta JA, Adelman A. Family practice patients' attitudes toward firearm safety as a preventive medicine issue: a HARNET study. Harrisburg Area Research Network. J Am Board Fam Pract 1999;12:354-9.

24. Monteith LL, Holliday R, Dorsey Holliman BA, Brenner LA, Simonetti JA. Understanding female veterans' experiences and perspectives of firearms. J Clin Psychol 2020;76:1736-53.

25. Simonetti JA, Dorsey Holliman B, Holiday R, Brenner LA, Monteith LL. Firearm-related experiences and perceptions among United States male veterans: a qualitative interview study. PLoS One 2020;15:e0230135.

26. Ramsawh HJ, Fullerton CS, Mash HBH, et al. Risk for suicidal behaviors associated with PTSD, depression, and their comorbidity in the US Army. J Affect Disord 2014;161:116-22.

27. Dobscha SK, Denneson LM, Kovas AE, Corson K, Helmer DA, Bair MJ. Primary care clinician responses to positive suicidal ideation risk assessments in veterans of Iraq and Afghanistan. Gen Hosp Psychiatry 2014;36:310-7.

28. Boggs JM, Quintana LM, Powers JD, Hochberg S, Beck A. Frequency of clinicians' assessments for access to lethal means in persons at risk for suicide. Arch Suicide Res 2020;1-10.

29. Naureckas C, Sacks CA, McGregor KA, Masiakos PT, Flaherty MR. Screening for access to firearms by pediatric trainees in high-risk patients. Acad Pediatr 2019;19:659-64.

30. Naganathan S, Mueller KL. Physician documentation of access to firearms in suicidal patients in the emergency department. West J Emerg Med 2019; 20:818-21.

31. Kroenke K, Spitzer RL, Williams JB. The Patient Health Questionnaire-2: validity of a two-item depression screener. Med Care 2003;41:1284-92.

32. Prins A, Bovin MJ, Smolenski DJ, et al. The primary care PTSD screen for DSM-5 (PC-PTSD-5): development and evaluation within a veteran primary care sample. J Gen Intern Med 2016;31:1206-11.

33. Hsieh HF, Shannon SE. Three approaches to qualitative content analysis. Qual Health Res 2005;15: $1277-88$.

34. Scientific Software Development GmbH. Atlas.ti. 2014; 7.1.8.

35. Office of Enterprise Integration (OEI), National Center for Veterans Analysis and Statistics (NCVAS). Profile of veterans: 2017. Available from: https://www.va.gov/vetdata/docs/QuickFacts/ 
2017_Veterans_Profile_Fact_Sheet.PDF. Accessed May 8, 2020.

36. Cleveland EC, Azrael D, Simonetti JA, Miller M. Firearm ownership among American veterans: findings from the 2015 National Firearm Survey. Inj Epidemiol 2017;4:33.

37. Siegel MB, Boine CC. The meaning of guns to gun owners in the US: the 2019 National Lawful Use of Guns Survey. Am J Prev Med 2020;59:678-85.

38. Valenstein M, Walters $H$, Pfeiffer $P$, et al. Acceptability of potential interventions to increase firearm safety among patients in VA mental health treatment. Gen Hosp Psychiatry 2018;55:77-83.

39. Lightfoot M, Comulada WS, Stover G. Computerized HIV preventive intervention for adolescents: indications of efficacy. Am J Public Health 2007;97:1027-30.

40. Rolen E. A closer look at veterans in the labor force. Bureau of Labor Statistics. 2017.

41. Butterworth SE, Anestis MD. Political beliefs, region of residence, and openness to firearm means safety measures to prevent suicide. Arch Suicide Res 2019;23:616-33.

42. Jordan JT, McNiel DE. Characteristics of persons who die on their first suicide attempt: results from the National Violent Death Reporting System. Psychol Med 2019;1-8.

43. Mertens B, Sorenson SB. Current considerations about the elderly and firearms. Am J Public Health 2012;102:396-400. 